\title{
Das Martyrium des Bischofs Cyprian.
}

\author{
Von P.Corssen in Berlin-Dahlem. \\ V. \\ Pontius.
}

Der literarische Charakter und die Tendenz der Schrift des

Mit dem Titel der Schrift des Pontius läßt uns die Überlieferung in Stich. Von den drei Handschriften Hartels ist die Überschrift in der ältesten unvollständig, die eine der beiden jüngeren bezeichnet die Schrift als eine kurze Lebensbeschreibung, die andere als passio beatissimi Cypriani martyris. Hieronymus nennt sie egregium volumen vitae et passionis Cypriani. Aber wir wissen nicht, ob er damit nur den Inhalt der Schrift oder ihren überlieferten Titel angeben will. Tatsächlich bildet das Leben und Leiden Cyprians, und da das Leiden nur ein Teil seines Lebens ist, sein Leben den Gegenstand der Schrift. Aber darum dürfen wir sie nicht ohne weiteres, wie Harnack, für eine Biographie erklären, denn nicht nur der Gegenstand, sondern auch die Behandlung kommt in Betracht, und wenn Harnack S. 36 hinzufügt, die Schrift sei zwar eine Biographie, aber lediglich unter dem Gesichtspunkt der opera und merita des Helden, so hebt er damit im Grunde sein Urteil wieder auf. Wir dürfen uns aber auch nicht auf eine Analyse der Schrift beschränken, sondern müssen versuchen, ihre Form aus der literarischen Geschichte zu erklären, und da uns vergleichbare Schriften aus der christlichen Literatur vor Pontius nicht zu Gebote stehen, so sehen wir uns auf die spätere christliche und die profane Literatur angewiesen.

Die beiden von Hieronymus angegebenen Teile der Schrift werden von Pontius selbst in der Einleitung unterschieden. Cyprian, sagt er, hat sich in seinen Schriften selbst ein Denkmal gesetzt. Aber er hat sich auch durch sein Leben Anspruch auf das Gedächtnis der Nachwelt erworben. Jedenfalls aber darf das Leiden eines solchen Mannes nicht mit Stillschweigen übergangen werden.

Aber vita steht bei Pontius nicht in dem umfassenden Sinne von Bíoc. Der griechische Christ würde dafür moגıteía sagen, Pontius 
selbst bestimmt den Begriff durch opera und merita, d. h. Werkheiligkeit, der die weiße Krone der Gerechtigkeit zum Preise wird. Das Leben in diesem Sinne beginnt natürlich erst mit dem Erwachen: des Glaubens. Daher schliebt Pontius alles, was vor der Bekehrung Cyprians liegt, von seiner Betrachtung aus. Schon daraus geht hervor, daß er keine Biographie im eigentlichen Sinne schreiben wollte, denn eine solche Beschränkung wird in den christlichen Biographien nicht geübt. Diese beginnen durchweg mit der fleischlichen Geburt ihrer Helden, auch wenn sie als Heiden geboren wurden, wie die Biographie Augustins von Possidius oder des Martinus von Sulpicius Severus. Pontius aber läßt sich durch den Zweck seiner Darstellung bestimmen, der mit Geschichte nichts $\mathrm{zu}$ tun hat, sondern in der Verherrlichung Cyprians besteht. Er will nicht an die dunkeln Schatten erinnern, die nach Cyprians eigenem Geständnis, auf seinem heidnischen Leben lagen: Daher kleidet er sogar das wenige, was er in der Form der Praeteritio über Cyprians rhetorische Studien mehr andeutet als aussagt, in ein Lob, ${ }^{1}$ obwohl selbst Augustin diese Studien als Teufelswerk verdammt. ${ }^{2}$ Er sagt uns nichts von der.Herkunft und der Heimat seines Helden, was doch die christlichen Biographen nicht minder wie die heidnischen für erwähnenswert halten, und der Schauplatz seiner kirchlichen Laufbahn wird ohne weiteres als bekannt vorausgesetzt.

$\mathrm{Zu}$ dem Leben Cyprians im weiteren Sinne, auch in dem beschränkten Zeitraum, den Pontius behandelt, gehört noch vieles andere; was Pontius als Biograph nicht hätte übergehen dürfen, was er aber deswegen nicht berührt, weil es zu seinem Zwecke nicht paßt. Eine Stelle ist für die Art seiner Darstellung besonders bezeichnend. Wir wissen aus Cyprians Briefen, daß seine Wahl zum Bischof gegen die Opposition von fünf Presbytern erfolgte. „Ich sage es ungern“, berichtet Pontius darüber, ;aber ich kann es nicht verschweigen: einige leisteten ihm Widerstand." Dieser Widerstand ist ein Mißklang zu den vollen Tönen, mit denen er den Enthusiasmus des Volkes preist. Ein solcher Mißklang hätte eigentlich in einem Leben, das Gott selbst zur Verherrlichung seines Trägers gestaltet hat, nicht vorkommen sollen.

1 Die Stelle ist fehlerhaft überliefert: c. 2 Fuerint licet (fuerint in licet $T$ ) studia et bonae artes devotum pectus imbuerint, tamen illa praetereo; nondum enim ad utilitatem nisi saeculi pertinebant. Man vermiBt ein Prädikat zu studia und die Be: ziehung auf Cyprian. Es wäre etwa zu erwarten: fuevint :ulilia studia et bonae artes, quae.

2 Sermo CCCXII In natali Cypriani IV \& 4. 
Daher fügte er sogleich hinzu: "damit er auch siegte", ${ }^{1}$ und deutet so den Widerstand in einen höheren Zweck um. Ohne Kampf kein Sieg. Wären nicht jene Widersacher gewesen; so wäre es eben kein Sieg gewesen. Von den Schwierigkeiten und Kämpfen aber, die Cyprian später gerade von diesen alten Widersachern bereitet wurden, verlautet kein Wort. Im Gegenteil, Pontius versichert, Cyprian habe ihnen verziehen und mit ihnen in vollster Harmonie gelebt.

Auf das sorgfältigste werden die gegen die Person Cyprians gerichteten, zum Schisma führenden Kämpfe in der karthagischen Gemeinde übergangen. Wohl wird der-kluge Steuermann gelobt, der das Schiff der Kirche glücklich durch die Wogen der Spaltungen hindurchführte, aber daß diese Spaltungen mit der persönlichen Bekämpfung Cyprians begannen und daß das novatianische Schisma, an das Pontius wohl insbesondere denkt, mit den Kämpfen in Karthago zusammenhing, davon läßt Pontius nichts ahnen. Nach ihm ist Cyprian nicht ein Teil der Ereignisse gewesen, er stand bändigend über ihnen, kaum bespritzt von dem tosenden Gischt.

Wird aber Cyprians Stellung zu dem novatianischen Schisma wenigstens angedeutet, so wird seine oppositionelle Kirchenpolitik gegen den römischen Bischof Stephanus überhaupt nicht berührt. Und doch erkennt der unterrichtete Leser den Standpunkt des Pontius an dem Beiwort pacificus, mit dem er den Nachfolger des Stephanus schmückt. ${ }^{2}$ Die Erinnerung an den Streit mit Rom, nachdem der Friede wiederhergestellt war, würde peinliche Empfindungen hervorgerufen haben und der gewünschte Eindruck von der Entwicklung des Lebens seines Helden dadurch gestört sein.

Außerdem gehört aber für Pontius alles das, worin wir die eigentliche Bedeutung Cyprians sehen, nicht $\mathrm{zu}$ den Werken und Verdiensten, die zu einem bevorzugten Platz im Himmel berechtigen. Nicht wegen seiner Haltung in Glaubensfragen, noch wegen der kraftvollen Verteidigung seiner bischöflichen Rechte und seiner Verdienste um den Ausbau der Hierarchie will Pontius Cyprian ein Denkmal setzen, sondern eben wegen der Heiligkeit und Werktätigkeit (c. 2), um derentwillen er schon als Neophyt zum Bischof gewählt wurde. Sein ganzes Leben lang übte Cyprian sich in der Nachahmung der Gerechten (c. 3). Bei der Bischofswahl trat auch seine Demut zutage (c. 5). In seinem Amte bewies er dieselben Tugenden, die er schon als Katechumene an

1 Uberliefert ist in $\mathrm{T}$ ut et vinceret, und das scheint mir richtig $z u$ sein (et vinceret $w \mu$, etiam ut vinceret v). Hartel schreibt et ut vinceret, was ich nicht verstehe.

2 Vgl. Tillemont, Mémoires IV 36. 
den Tag gelegt hatte. Daneben zeigte er Tatkraft und Strenge, gepaart mit Huld. Der Hauptnachdruck aber wird auch hier auf seine Fürsorge für die Armen gelegt (c. 6).

Diesen Verdiensten wurde der Lohn in dem stufenweisen Aufstieg zum Ruhme des Bekenners und Märtyrers zuteil, der durch die Ächtung (c. 7), die Verbannung (c. 11-13) und das Martyrium (c. 14ff.) führte.

Aber die beiden so sich ergebenden ungleichen Teile, c. 2-6 und 7-18 mit dem Schlußkapitel 19, werden untereinander' ausgeglichen und miteinander in Verbindung gehalten durch eine chronologische Anordnung des Stoffes, so daß der Höhepunkt in der Liebestätigkeit Cyprians erst mit der Schilderung seines Wirkens während der Pest im zweiten Teile c.9f. erreicht wird, wobei der Leser durch die Wiederholung einer c. 7 angewandten Übergangsform ${ }^{1}$ über die in der $\mathrm{Er}$ zählung klaffende Lücke von. $252-257$ hinweggetäuscht wird. Übrigens wird das chronologische Prinzip nicht streng eingehalten, sonst würde c. 3 seinen Platz hintér c. 5 gefunden haben. . Der. Abschluß aber des ersten Teils durch die züsammenfassende Charakteristik Cyprians (c. 6) zeigt eindringlich die Vorherrschaft des: sachlichen.Prinzips .vor dem chronologischen. Einzelne bestimmte Zeitangaben, wie sie sich auch in christlichen Biographien finden, fehlen völlig. Die Zeit der Ereignisse zu bestimmen, hat ebensowenig in der Absicht des Pontius gelegen wie den Ort. Beides wird als bekannt vorausgesetzt. . Würde nicht beiläufig das Martyrium des Bischofs Sixtus in Rom erwähnt (c. 14), so würde Pontius die Zeit Cyprians völlig im Dunkel gelassen haben.

Vergleichen wir aber den Ton der Schrift des Pontius mit dem der wirklichen christlichen Biographien, so kann ihre. Wesensverschiedenheit unmöglich verkannt werden. Nehmen wir z. B. das Leben des hl. Martinus von Sulpicius Severus. Sein Werk hat denselben Zweck wie das des. Pontius, und er spricht inn wie dieser aus. Er will das Leben des Martinus erzählen als ein Vorbild echt christlichen Wandels zur Nacheiferung der Leser $(1,6)$. Aber er läßt die Tatsachen für sich selber sprechen. Flicht er ein lobendes Wort ein, so geschieht es, ohne daß er die Erzählung unterbricht. Selten erhebt er sich zu einem bewundernden Ausruf wie 10,9 oder 25,6. Die Schrift des Pontius wimmelt von rhetorischen Fragen: „Wer hat jemals etwas so Wunderbares erlebt?" "Wer, bitte, von den Alten hat das fertig gebracht?"

1 c. 7: Statim denique pro talibus meritis etiam proscriptionis gloriam consecutus est. c. 11: His tam bonis et tam piis actibus superventit exiltum. 
(c. 2). „Wer sollte einem mit solchem Gemüte Glaubenden nicht die Berechtigung $\mathrm{zu}$ allen Ehrenstufen glauben?" (c. 3) usw. Pontius erzählt nicht einfach, er fügt überall sein Urteil hinzu. Er versichert in immer neuen Wendungen, wie beispiellos und einzigartig die Tugenden seines Helden waren. Er sucht die Wirkung nicht sowohl durch die Dinge selbst als durch seine Darstellung zu erreichen. Kurz, es ist der Ton des Panegyrikers, den er von der ersten bis zur letzten Zeile festhält.

Dieser Ton stimmt zu dem Zweck der Schrift, nicht sowohl von einzelnen Lesern für sich gelesen als von einer versammelten Gemeinde gehört zu werden. Dagegen kanin nicht ernsthaft eingewendet werden, daß der Verfasser selbst von der Schrift als von einem Buche redet (c. 10), und zwar einem Buche, das nicht nur für die Gegenwart, sondern auch für die Nachwelt bestimmt ist (c. 1). Es gibt Reden, die mehr oder. weniger extemporiert, und Reden, die vorher sorgfältig ausgearbeitet sind, Reden, die aus dem Gedächtnis frei vorgetragen, und Reden, die abgelesen werden, Reden, die noch so sehr aus der Stunde geboren sein mögen und ihre Bedeutung nur in der Stunde haben und doch von ihren Verfassern als ein opus aeve perennius in Buchform der Ewigkeit übergeben werden. Pontius hat zunächst für einen ganz bestimmten Kreis bei einer bestimmten Gelegenheit geschrieben - bei welcher, werden wir noch $\mathrm{zu}$ untersuchen haben - und das Geschriebene zur Verlesung gebracht. Zu diesem seinem Publikum steht $\mathrm{er}$ in den engsten Beziehungen, die Hörer kennen ihn und glauben ihm, und wiederum vertraut er ihnen. Er kann von ihnen sagen, daß sie wissen, er sei bereit gewesen, mit Cyprian zu sterben (c. 19). Sie wünschen von ihm alles über Cyprian zu hören. Vos nos auribus fatigatis, sagt er mit einer unübersetzbaren Wendung, um das unersättliche Interesse seiner Zuhörerschaft auszudrücken. - Es kann kein Zweifel sein, die Schrift ist als Rede gedacht und zum Vortrag bestimmt gewesen, wenn auch die Hörer nicht näher bezeichnet sind. Ist sie nun nicht als eine Lebensbeschreibung, sondern als eine Lobrede anzusehen, so bleibt die Frage, wie die Einführung dieser Gattung in die christliche Literatur $z \mathbf{u}$ erklären ist, ob. sie auf der Übertragung profaner Vorbilder beruht oder ob sie in anderen und in welchen Umständen $\mathrm{zu}$ suchen ist.

Nach Reitzenstein hat Pontius den Gedanken und die Form seiner Schrift der Literatur der exitus illustrium virorum entlehnt (S. 52), die ihm die beiden, in einem jeden Stücke dieser Gattung nötigen Teile geliefert habe (S.53). Diese Literatur blühte unter Trajan. Der jüngere 
Plinius, der zwei Werke dieser Art nennt, vergleicht sie mit Leichenreden. Die Gefeierten waren eines gewaltsamen Todes gestorben als Opfer der Tyrannenwillkür. In dem einen Falle sagt Plinius es ausdrücklich (ep. 5, 5, 3), in dem andern deutet er es verständlich an (ep. 8, 12, 4). Unleugbar sind also die allgemeinen Voraussetzungen, unter denen diese Literaturw.erke entstanden, denen vergleichbar, die der Schrift des Pontius zugrunde liegen. Allein damit ist ein literargeschichtlicher Zusammenhang zwiśchen beiden keineswegs bewiesen, denn die profane Gattung war durch ihren Gegenstand auf eine bestimmte Zeit beschränkt, und es lief keine lebendige Tradition von der trajanischen Zeit bis auf die Mitte des 3. Jahrhunderts hinab, deren Zwang auf Pontius hätte wirken können. ${ }^{1}$ Es ist fraglich, ob jene Werke und ihre Vorläufer aus der Zeit Cäsars, die laudes Catonis, ${ }^{2}$ im 3. Jahrhundert etwa in den Rhetorenschulen noch gelesen wurden. Aber selbst wenn es der Fall war, so sprechen die Lebensumstände des Pontius, soweit sie sich erkennen lassen, nicht dafür, daß Pontius einen Einfluß, von dieser Seite empfangen hatte, die Analyse seiner Sprache und seines Stils sogar dagegen." Da nun aber jene Werke in ihrem Wesen offenbar nichts anderes als eine besondere literarische Abart der oratio funebris waren, diese aber zu Pontius' Zeit sicher noch durchaus lebendig war, so könnte man vielleicht an eine Einwirkung von daher denken. Allein auch mit einer solchen Annahme wäre die Entstehung der Schrift des Pontius nicht erklärt. Denn bei den Leichenreden handelte es sich eben nicht um den besonderen Fall, der den Vergleichungspunkt bildet, den jene Literaturwerke der trajanischen Zeit mit ihr gemeinsam hatten. Ferner waren die Leichenreden nicht literarisch, und Pontius wollte doch ein Literaturwerk schaffen. Lassen sich daher keine profanen Anregungen ermitteln, die es hervorgerufen hätten, so werden wir die. Bedingungen seiner Entstehung schon in den kirchlichen Verhältnissen der Zeit und den persönlichen des Pontius suchen müssen. Da nun die Schrift trotz ihrer literarischen Ansprüche ihren Charakter als Gelegenheitsschrift nicht verleugnet, so werden wir am besten den Weg einschlagen, daß wir die Gelegenheit zu ermitteln suchen, bei der Pontius sein Werk der christlichen Versammiung, zu der er spricht, vorgetragen hat. $\mathrm{Zu}$ diesem $\mathrm{Zweck}$ müssen wir zunächst die Tendenz der Schrift scharf erfassen.

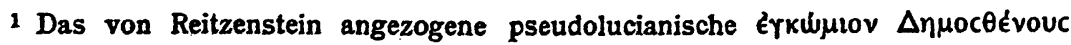
steht doch wohl jener Literatur ziemlich fern und scheint mir keine Analogie zu der Schrift des Pontius zu sein.

2 Vgl. Peter, Die geschichtliche Literatur über die römische Kaiserzeit I 186. 
Es kommt Pontius darauf an, die zwiefache Bedeutung Cyprians, des glorreichen Priesters und Zeugen Gottes, darzustellen. Durch seine Werke verdiente sich Cyprian das Bischofsamt, und als Bischof wirkte er vorbildlich durch seine Werke. Den Gerechten des alten Bundes tat er es gleich (c.3), ja übertraf sie (c. 10). Er war der vollkommene Gerechte und der. vollkommene Märtyrer. Er wurde nicht. erst durch sein Martyrium vollkommen, er war. es bereits durch seine Werke. Der Gerechte lehrt durch sein Leben, der Märtyrer durch seinen Tod. Cyprian tat beides. Er war ein Lehrer auch ohne das Martyrium. Das unterschied ihn von der großen Masse der Märtyrer, die außer ihrem Martyrium keinen Ruhmestitel hatten und durch ihren Tod oft ein höchst fragwürdiges Leben krönten. Aus der Vergleichung des bischöflichen Märtyrers mit den martyres plebei et catechumeni spricht nicht nur klerikaler Dünkel und klerikaler Parteistandpunkt, obwohl man beides nicht unterschätzen darf, sondern auch eine Auffassung des Martyriums, wonach es nicht schlechthin der höchste Beweis christlicher Gesinnung ist. Diese Auffassung hat Pontius von Cyprian gelernt. Er scheut sich nicht, den Fall zu setzen, daß der Herr, wie das erste Mal während der decianischen Verfolgung, so auch das zweite Mal während der valerianischen. Cyprian das Martyrium versagt hätte (c. 14). Ein. Rigorist vom Schlage des Verfassers der aus Cyprians Zeit stammenden Schrift „De laude martyrii“, wäre schwerlich auf einen solchen Einfall gekommen. Aber mit dieser gemäßigten Auffassung des Martyriums ist doch die andere eng verbunden, daß erst das Martyrium die Krone des Lebens verleihe, und die tiefe Genugtuung, daß Cyprian, dem .anfangs die Krone vom Herrn versagt zu sein schien, sie schließlich doch errang (c. 19), ist unverkennbar. Ja, das Besondere und Eigenartige in der Schrift des Pontius besteht gerade in der Verschmelzung dieser beiden Ideale, und zwar in der Person des Priesters. Aber in dieser nicht völlig ausgeglichenen Anschauung überwiegt doch die alte Vorstellung von der unbedingten Herrlichkeit des Martyriums, und so erscheint Pontius das Leben Cyprians von vornherein auf dieses angelegt. Als das Volk so dringend Cyprian zum Bischof verlangte, erkannte es, wie der Ausgang bewies, ahnungsvoll in ihm den prädestinierten Märtyrer.(c. 5). Nach Gottes Ratschluß wurde er stufenweis zu dem Ruhme des Martyriums emporgeführt (c. 7). So sind die beiden Teile, in welche die Schrift zerfällt, nicht einander gleichwertig, sondern der erste ist nur die Voraussetzung des zweiten. Die Lobrede gipfelt nicht nur in der Passion, sondern es ist recht eigentlich die Passion, die gefeiert werden soll, und nur darum wird 
auch das Leben behandelt, weil erst dadurch diese-Passion in ihr besonderes, sie von anderen unterscheidendes Licht tritt. Pontius gibt sich die größte Mühe, seine Darstellung so zu gestalten, daß Cyprians Passion als eine Fügung Gottes und nicht als ein Spiel des Zufalls erscheint. Darum legt er dem Traum Cyprians in Curubis, durch den ihm nach der Deutung, die man ihm nach seinem Tode gab, Jahr und Tag seines Martyriums vorausgesagt war (c. 12; vgl. XVI 226ff.), eine so hohe Bedeutung bei. Es wird geschildert, wie in dem ganzen Verlaufe des Martyriums der Finger Gottes sichtbar hervortrat. Die göttliche Güte gewährte es Cyprian, daß das Volk bei seiner Passion wachte, als er die Nacht im Hause des Princeps zubrachte, während die Jünger, so soll man in Gedanken ergänzen, im Garten von Gethsemane schliefen. Es war keine Laune des Prokonsuls, daß das Martyrium um einen Tag aufgeschoben wurde, sondern das geschah zufolge des Gesichtes, mit dem er vor einem Jahre begnadet war (c. 15). Das Urteil des Prokonsuls war, ihm unbewußt, ein göttliches Orakel (c. 17). ${ }^{1}$ Auch das war göttliche Fügung, daß seine Anhänger auf dem Richtplatz auf die Bäume stiegen, denn damit wiederholte sich, was Christus von Zachäus widerfahren war. Der Henker aber konnte den tödlichen Streich nicht führen, ehe nicht sein Arm vom Himmel die Kraft dazu empfangen hatte (c. 18).

So liegt der Schwerpunkt der Schrift durchaus in der Passion Cyprians. Auf die besondere Gelegenheit aber, für welche die Schrift bestimmt war, wirft der Hinweis auf die Passion gewöhnlicher Christen in der Einleitung Licht. Es ist schon gesagt worden, daß dabei an die Passio Perpetuae gedacht ist (S. 138). Die Passio Perpetuae ist etwas in ihrer Art durchaus Neues, und der Verfasser ist sich dessen wohl bewußt. Sie beruht zum größeren Teil auf den Aufzeichnungen der Märtyrer selbst. ${ }^{2}$ Die Fragen und Antworten in dem Verhör des

1 Ich benutze die Gelegenheit, eine von mir XV 316 gegebene falsche Interpretation dieser Stelle zu berichtigen. Man darf sich weder durch den Plural pontifices, noch, wie Weyman a. a. O. Sp. 1279 sich richtig ausdrückt, durch den rhetorisch übertreibenden Gebrauch von solere täuschen lassen. Pontius denkt nicht, wie ich meinte, an heidnische Priester, sondern an den Hohenpriester Kaiphas, Joh. 11, 51, eine Stelle, auf die Harnack S. 28 richtig hingewiesen hat. Wie Kaiphas das Leiden Jesu, so sagte der Prokonsul das der Cyprian nachfolgenden Märtyrer voraus. Wunderlich bleibt nur, dab Pontius den Prokonsul zum Pontifex macht, denn nach der Handschrift $T$, der Hartel und auch Harnack folgen, tut er das. Aber das ändert sich, sobald man auf die Seite der jüngeren $\omega \mu$ tritt: cum soleant de passione pontifices etiam prophetare. Nun kommt die richtige Begründung heraus: warum sollte der heidnišche Prokonsul nicht prophezeien, da es auch der jüdische Hobepriester tat?

2 Das scheint mir aus sprachlichen und stilistischen Gründen sicher. 
Prokonsuls, die in den Acta Scillitanorum den Inhalt erschöpfen, werden darin nur flüchtig gestreift, dagegen die Visionen der Märtyrer im Gefängnis und ihre Passion selbst ausführlich geschildert. Der Verfasser ist ein rhetorisch geschulter Mann. Als fürchte er, daß ihm die einfache Rede der Märtyrer, denen er von c. 4 bis 13 das Wort läßt, keine Gelegenheit biete, dies zu zeigen, hat er eine besondere stilistische Kunst auf die Einleitung verwendet und gleich die erste Periode mit einem wahren Raffinement gebaut. Die Passion ist im ganzen schlicht und einfach erzählt, aber auch hier ist nicht ganz auf rhetorischen Effekt verzichtet. Hin und wider wird eine Reflexion eingeflochten. Am Schluß bricht der Verfasser in laute Bewunderung aus: $O$ fortissimi ac beatissimi martyres! o vere vocati et electi in gloriam domini nostri Iesu Christi! Aber innerhalb der Erzählung fehlen solche Ausrufe. Der Verfasser sagt in der Einleitung und am Schlusse, zu welchem Zweck er die Passion der Perpetua und ihrer Genossen aufgeżeichnet hat. Als Montanist ist er der Überzeugung, daß die Wirkung des Geistes dem Wechsel der Zeiten nicht unterworfen ist, ja daß sie in der Gegenwart sich größer erwiesen hat als in der Vergangenheit. Daher ist das Vorbild der jüngsten Märtyrer nicht weniger wertvoll als die alten Vorbilder, und die Gnadenwirkung des Geistes in ihnen muß aufgezeichnet und zur Erbauung der Gemeinde gelesen werden.

Damit wird verlangt, daß die Passionen wie die heiligen Schriften des AT und NTs im Gottesdienste verlesen werden. Hieraus aber folgt, daß die kurzen Märtyrerakten, wie die Acta Scillitanorum nicht zum Vorlesen bestimmt waren und daß ihre Art dem Verfasser der Passio Perpetuae zur Ehrung der Märtyrer nicht zu genügen schien. Es ist nicht ausdrücklich ausgesprochen, aber es ist selbstverständlich, daß nach der Absicht des Verfassers die Passio an dem Jahrestage des Martyriums verlesen werden sollte. Dieser Gedanke ist aber nicht unmittelbar nach dem Martyrium entstanden. Die Passio Perpetuae ist erst geraume Zeit nach dem Martyrium, wenn auch vor Tertullians De anima (s. c.55), verfaßt worden. Denn der Verfasser wendet sich an die Älteren, die das Martyrium selbst miterlebt haben, und die Jüngeren, die es erst durch seine Aufzeichnungen kennen lernen (c. 1 g. E.). Dazu stimmt, daß von dem Mädchen, das von Felicitas zwei Tage vor dem Martyrium geboren wurde (c. 15), gesagt wird, eine Schwester habe es sich zur Tochter aufgezogen. Gefeiert wurde der Jahrestag des Martyriums natürlich schon früher, und es ist sehr wahrscheinlich, daß es auch kurze Akten davon in der Art der Scillitanerakten gab. 
Obwohl die Schrift des Pontius keineswegs eine Parallele zu der Passio Perpetuae ist wie die Passio Montani et Lucii, die in bewußtem Wetteifer mit ihr geschrieben ist, so befand sich Pontius doch seinem Gegenstande gegenüber in einer ähnlichen Lage wie der Verfasser der Passio Perpetuae dem seinen. Die den einfachen Tatbestand des Martyriums schlicht erzählenden Akten genügten ihm nicht. Er hätte sie aber schwerlich so völlig ignorieren können, wenn sie schon damals an dem Jahrestage des Martyriums vor der Gemeinde verlesen worden wären, wie es später mit solchen Akten geschah, eine Sitte, die wohl erst im Laufe des vierten Jahrhunderts entstanden ist. Das Martyrium aber eines Mannes wie Cyprian schien ihm dringend nach einem würdigen Denkmal zu verlangen. Da nun das Verhör vor dem Prokonsul und die Vorgänge des Martyriums selbst dazu keinen hinreichenden Stoff boten, so schlug er einen andern Weg ein, seinen Helden zu feiern, indem er sein.Leiden als das gottgewollte Endresultat seines ganzen Lebens darstellte. Trug er sein Werk, wie er andeutet, selbst der Gemeinde vor, so kann das kaum an einem andern als dem Gedächtnistage des. Martyriums gewesen sein. So haben wir die Schrift des Pontius in die Passionenliteratur einzureihen, als eine im Anschluß an die Passio Perpetuae, aber auch im Gegensatz $\mathrm{zu}$ ihr entstandene neue Form der Passio.

Diese Auffassung bestätigt sich, wenn wir uns nach der Wirkung der Schrift umsehen. Sie tritt besonders deutlich in zwei Passionen der Donatistenzeit hervor, der Passio Marculi und der Passio Maximiani et Isaac. ${ }^{1}$

Die Verfasser beider sind mit der Schrift des Pontius genau bekannt und schreiben unter ihrem Einfluß. Beide betrachten das neue Moment in ihr, die Berücksichtigung auch des Lebens der Märtyrer, als selbstverständlich. Et quia praetermittendus non est superioris vitae memorabilis cursus, heißt es in der einen Passio, ${ }^{2}$ de virtutibus Marculi gloriosi, etsi propter multitudinem nequeunt omnia, ${ }^{3}$ propter testimonium tamen vel pauca. dicenda sunt. Dieses Wenige wird allerdings mit einer verblüffenden Kürze abgemacht. Von dem Leben des Märtyrers wird so gut wie nichts gesagt, aber es wird genau unter denselben Gesichtspunkt gestellt wie das Leben Cyprians bei Pontius: Beweis für die Vortrefflichkeit seines Lebens ist seine Wahl zum Bischof, für die tadel-

1 Nach Mabillon, Analecta t. IV abgedruckt bei Migne P. L. 8, $758 \mathrm{ff}$. u. $767 \mathrm{ff}$.

2 P. L. 8, 760D.

3 Vgl. Pontius c. 6 XCV 7 enumerare cuncta eius onerosum est. 
lose Führung seines Bischofsamtes sein Martyrium. ${ }^{1}$ Auch der Verfasser der Passio Maximiani et Isaac erkennt die Forderung an, daß nicht nur das Leiden, sondern auch das Leben des Märtyrers geschildert werden müsse, obwohl er sich dieser Forderung durch die Erklärung entzieht, daß beides zu vereinigen unmöglich sei: Si vitae totius memorare mores incipiam, revertenti martyrio facere videbor iniuriam; ad praedicationem martyrii si gressus dirigam, tantae vitae contemptor tenebor adstrictus. ${ }^{2}$ Aber durch die Aufstellung dieses Dilemmas tritt die Anerkennung des Prinzips um so deutlicher hervor. Der Verfasser dieser zweiten Passio hat die Form eines Briefes an numidische Gemeinden gewählt, aber daß sie für den Jahrestag des Martyriums bestimmt ist, zeigt der Satz revertenti martyrio facere videbor iniiuriam.

Wie die Entwicklung von der Passio Perpetuae über die Schrift des Pontius zu der Passio Marculi gegangen ist, scheint mir recht deutlich aus der gleichartigen Motivierung ihrer Arbeit bei allen drei Verfassern hervorzugehen. Der Verfasser der Passio Perpetuae beruft sich auf die Verherrlichung der alten Glaubenshelden, Pontius auf die Passio Perpetuae, der Verfasser der Passio Marculi will die Opfer der Traditoren den alten Märtyrern gleichgestellt wissen (Iisstum ac satis dignum est, ut antiquorum testium laudibus recentium quoque martyrum virtus adiuncta glutinetur). Die Ausrufe am Schlusse der beiden donatistischen Passionen ( $O$ memorabile $b$. Marculi atque insigne martyrium! $O$ appetendum omnibus inconcussae virtutis exemplum! und $O$ beatae passionis memorabilis gloria, o felix exitus) erinnern an den Schluß der Passio Perpetuae (s. oben S. 210). Aber die ganze Art der Darstellung mit ihrer von dem schlichten Tone der alten Märtyrerakten so völlig abweichenden Rhetorik ist durchaus der Schrift des Pontius nachgebildet. Einzelne Wendungen kehren wörtlich oder wenig verändert wieder, so z. B. 8,760 D suspensa ad caelum mente $=$ CVI 1. 8,769 A fecundum pectus $=$ XCVII 9. 8,767 C praepropera celeritate $=$ XCII 19 praepr. velocitate. 8, $761 \mathrm{C}$ hic... enarrare quis sufficiat? 8, 769 A

1 P. L. 8,761 A quae vero in illo fuerit probitas conscientiae, non arbitror dine multhumque laborandum, cum superiorem eius vitam illa res probat, quod meruit sacerdotium; sacerdotizm vero qualiter gesserit, inde fit clarnm, cui pro praemio dominus dignatus est praestare martyrizum. Pontius XCV 8 ad probationem bonorum operum solum hoc arbitror satis esse, quod ad officium sacerdotii electus est. c. 7 XCVII 5 pro talibus meritis etiam proscriptionis gloriam consecutus est. Posset quidem tum etiam marlyrii circa eım debila corona properare, nisi usw.

2 P. L. 8, 767 C. 
exhinc referre quis possit? $=\mathrm{XCVI} 9$ exinde . . quis referre sufficiats 8,767C unde igitur incipiam: $=\mathrm{XCI} 16: 8,769 \mathrm{~A}$ sed quid pluribus? = XCVIII 9 quid plura? Ganz im Geiste des Pontius werden auch die einzelnen Vorgänge aufgefaßt. Auch diese Märtyrer werden vorher ihres Martyriums durch eine Vision versichert. Dabei wird die Deutlichkeit und Zuverläsșigkeit der Vision und ihre beruhigende Wirkung ganz wie bei Pontius hervorgehoben: Neque enim aliquid est, quod obscurum aut ambigum in hac revelatione videatur,... in qua meruit et palmam, per quam victoriam sperans esset iam de congressione securus heißt es von der Vision des Marculus (M. X 763 B) und bei Pontius: antistitem suum esse voluit de secutura passione securuin (c. $12 \mathrm{CIII} 1$ ). und nach der Erzählung der Vision: Quid hac revelatione manifestius? (c. 13 CIV 5). Diese Frage kehrt in der Passio Maximiani et Isaac bei der Vision des Isaac fast wörtlich wieder: Quid hac visione manifestius: $(8,771 \mathrm{~B})$, während der andere Zug auf den ebenfalls mit einer Vision begnadeten Maximianus übertragen ist: de tali securitate iam fretus (8, 769A). Wie Cyprian und seinen Nachfolgern der Prokonsul, so wird Marculus im Gefängnis der Henker zum Propheten, wobei ebenso wie bei Pontius auf Joh 11,51 hingewiesen wird. ${ }^{1}$ In der Schilderung: Egrediebatur de domo Marculus gloriosus, custodum excubiis ac militum classe circumdatus... egrediebatur vultu laetus $(8,764 \mathrm{C})$ erkennt man deutlich das Vorbild wieder. ${ }^{2}$ Der Verfasser der Passio Maximiani et Isaac läßt sich sogar die dilatio unius diei und den dies alter nicht entgehen, die bei Pontius eine so große Rolle spielen. Auch hier wird, sehr gezwungen, der Aufschub, der darin besteht, daß der Tod des Maximianus einen Tag nach dem des Isaac erfolgt, als eine göttliche Fügung ausgedeutet. Denn als das Volk das. Verscheiden Isaacs hörte, strömte es herbei, um sich in den Besitz der Leiche zu setzen, und als ihm diese verweigert wurde, wachte es die ganze Nacht, wie bei Cyprian, als er in dem Hause des Princeps lag. Das war die Ehre, die der Herr dem Märtyrer verschaffen wollte. Eine weitere Parallele ist bereits oben S. 129 aufgeführt.

So dürfen wir diese beiden Passionen, die so ganz in den Spuren des Pontius wandeln, wohl als eine Bẹtätigung unserer Auffassung von dem Charakter seiner Schrift ansehen. Sie bezeugen zugleich die hohe Bedeutung, die diese Schrift für das Fortleben des Andenkens an Cyprian in der donatistischen Kirche gehabt hat.

1 Vgl. oben S. 209.

2 Vgl. oben S. 138 und CVII 22 egressus est domum principis, CVIII 27 cum exiret praetorii fores, ibat comes militum turba. 
Aber ihre Nachwirkung in der katholischen Kirche Afrikas ist nicht minder groß gewesen. Sie läßt sich deutlich in den Predigten Augustins über das Martyrium Cyprians wahrnehmen. Augustin hat der ersten dieser Predigten zwar die Cypriansakten zugrunde gelegt, aber er interpretiert sie ganz in Pontius' Geist. An mehreren Stellen hat er auch direkte Anleihen bei ihm gemacht. Serm. CCCIX 2 Quod pro fide confessionis in exilium Curubim missus est, non s. Cypriano aliquid nocitum, sed multum illi praestitum est civitati = CIII 1 ut non exulem tantummodo Curubis, sed et martyrem possideret. Seine Betrachtungen über die Bedeutung des Exils für einen Christen sind dieselben wie bei Pontius: ibid. $O$ stulta infidelitas... de patria sua in alienam te arbitraris excludere hominem dei, in Christo nusquam exulem, in carne ubique peregrinum = XI 13 Sed viderit saeculum, cui inter poenas exilium putatur...18 admixtus dei sui rebus exilium non potest computare; adde quod deo integre serviens etiam propria in civitate peregrinus est. Von Pontius hat er die Bemerkung übernommen, da $\beta$ Cyprian durch die plötzliche Verhaftung nicht überrascht worden sei CCCIX, 3 Quid iam fremeret persecutoris impetus adversus cor semper paratum accedente etiam domini revelatione firmatum? $=$ CVI 18 Unde posset tamquam improviso impetu mens semper parata subitaris Auch die bis zur Geschmacklosigkeit gesteigerte Überschwenglichkeit, mit der Augustin von der Schwierigkeit der Aufgabe spricht, dem Verdienste eines Märtyrers wie Cyprian gerecht $\mathrm{zu}$ werden, ist sichtlich von Pontius inspiriert: Sermo CCCXIII 1. Cuius reverendi episcopi et venerandi martyris (vgl. XC 3 antistes ac testis dei) laudibus nulla lingua sufficeret nec si se ipse laudaret. In hoc itaque sermone nostro, quem de illo debitum vestris auribus reddimus, ${ }^{1}$ magis approbate voluntatis affectumn quam exigite facultatis effectum. Vgl. XCI 11 In qua parte si dixero nos opibus facundiae defici minus dico; facundia enim ipsa deficitur digna facultate. Sermo CCCXII, 1 si quid minus quain debetur exsolvero, non me despiciat loquentem. Vgl. XCII, 1 hanc petens veniam, ut quicquid minus dixero, ignorantiae meae derogetur.

Wenn Pontius mit seinem Bestreben, das Leben und Leiden seines Helden mit einer Gloriole zu umgeben, bei der Nachwelt Glück gehabt hat, wenn er bemüht gewesen ist, alle Feindseligkeiten und Widerstände, mit denen Cyprian zu kämpfen gehabt hatte, aus seiner Darstellung auszuschalten, so läßt sich gleichwohl nicht verkennen, daß mit der panegyrischen Tendenz in seiner Schrift eine apologetische

1 Vgl. oben S. 206. 
eng verbunden ist, die durch die laute Überschwenglichkeit des Lobes mehr verraten als verborgen wird. Diesè apologetische Tendenz ist von uns bereits mehrfach berührt worden, wir müssen sie nun planmäßig verfolgen.

Zwei Umstände im Leben Cyprians boten vor allem den Angriffen eine Zielscheibe, die Art seiner Erwählung zum Bischof und seine Flucht in der decianischen Verfolgung. Über den ersten Umstand gibt uns Pontius eine unschätzbare Aufklärung. Wir erfahren durch ihn, daß Cyprian sofort nach seiner Taufe, ehe er irgendein kirchliches Amt bekleidet hatte, zum Bischof gewählt wurde, und zwar ohne Assistenz von andern Bischöfen. Diese Tatsache ist freilich bis heute nicht deutlich erkannt. Benson bringt es fertig, Cyprian vom Diakonen durch das Presbyterat zum Bischof aufsteigen zu lassen, während Harnack meint, er scheine die niederen Grade übersprungen zu haben, sei aber Presbyter gewesen (S. 9). Der Irrtum ist dadurch entstanden, daß Pontius allerdings an einer Stelle von Cyprian als Presbyter spricht, aber man darf diese Stelle nicht äußerlich und ohne Rücksicht auf den $\mathrm{Zu}$ sammenhang betrachten, aus dem ersichtlich ist, daß presbjter hier nicht im Unterschied von episcopus gesetzt ist.

Ganz unmißverständlich sagt Pontius c. 5, daß Cyprian gleich nach seiner Taufe zum Bischof gewählt sei: iudicio dei et plebis favore ad officium sacerdotii et episcopatus gradum adhuc neophytus et ut putabatur novellus electus est. Wer annimmt, daß Cyprian Presbyter war, bevor er Bischof wurde, ist gezwungen, sacerdotium in einem andern Sinne als episcopatus zu nehmen. Es kann allerdings in einem weiteren Sinne stehen, denn auch die Presbyter sind sacerdotes. Aber steht das Wort in diesem allgemeineren Sinne, so dient es eben nicht zur Unterscheidung. Auch hier kann es gar nicht zur Unterscheidung dienen, denn wäre Cyprian erst Presbyter geworden, so wäre er, wenn er zum Bischof gewählt wurde, nicht mehr Neophyt gewesen. Es ist aber auch in der ganzen Beschreibung nur von einem Akt die Rede, der Bischofswahl. Pontius braucht eben sacerdotium und sacerdos synonym mit episcopatus und episcopus, wie es Cyprian unzählige Male tut. "Das Volk verlangte nicht nur nach einem Priester, sondern auch nach einem zukünftigen Märtyrer", heiBt es XCV 22: non solummodo sacerdotem, sed et futurum martyrem requirebat. Unmittelbar vorauf geht Z. 20 concupiscens, ut exitus docuit, non episcopum tantum. Wie hätte aber auch von dem Volke, das Cyprian zum Bischof wählen wollte, gesagt werden können, es habe nicht nur nach einem Priester verlangt, wenn er schon Presbyter gewesen wäre? Die Erzählung von der 
Bischofswahl in c. 5 steht aber keineswegs in Widerspruch mit c. 3, denn auch hier wird nun davon gehandelt, daß Cyprian sogleich die höchste Stelle in der Gemeinde errang. Darum setzt sich Pontius mit der apostolischen Vorschrift auseinander, daß Neophyten nicht zum Bischofsamt zugelassen werden sollen. Bei Cyprian fiel der Grund fort, der $\mathrm{zu}$ dieser Vorschrift geführt hatte. Denn sein Glaube war von vornherein so stark und so erleuchtet, daß jeder ihn sofort aller Stufen der kirchlichen Rangordnung für würdig erklären mußte: Quis enim non omnes honoris gradus crederet tali mente credenti? Wenn hier unmittelbar voraufgeht: presbyterium vel sacerdotium statim accepit, so ist sacerdotium erläuternd $\mathrm{zu}$ presbyterium hinzugesetzt, damit dieses nicht im engeren Sinne gegensätzlich $z \mathbf{u}$ episcopatus verstanden werde. Danach sagt er dann in demselben Sinne: multa sunt quae adhuc plebeius, multa quae iam presbyter fecerit. Die Antithese zu presbyter bildet hier lediglich plebeius, nicht aber zugleich episcopus. Es soll zwischen dem Laien und dem Geistlichen, und zwar dem Geistlichen im eigentlichen Sinne, unterschieden werden, denn presbyter schließt die niederen Grade der dienenden Geistlichen aus, aber den des Bischois ein. Auch der Bischof ist Presbyter, und obwohl gerade Cyprian seine höhere Würde als Bischof gegenüber den Presbytern ganz besonders betont hat, so ist doch auch aus seinem Sprachgebrauch die Erinnerung an das alte Verhältnis noch nicht völlig geschwunden, das in der Bezeichnung der Presbyter als compresbyteri von seiten des Bischofs ausgedrückt ist (z. B. Ep. 1, 1. 14, 4. 71, 1). Ausdrücklich wird die Wesensgleichheit des Presbyters und Bischofs Test. III $8 \dot{5}$ anerkannt, da hier das Gebot, daß man vor dem Bischof oder Presbyter aufstehen soll, auf Lev 19, 32 gestützt wird, wo natürlich ausschließlich von dem Presbyter die Rede ist. Wie stark aber das Bewußtsein von ihrer alten Bedeutung noch in den Presbyterien selbst war, mußte gerade Cyprian an sich erfahren. Die römischen Presbyter scheuten sich nicht, nach seiner Flucht in seine Machtsphäre einzugreifen, und sehr bald maßten sich dann Presbyter seiner eigenen Gemeinde Befugnisse an, die er einem unter seiner Leitung stehenden Konzil vorbehalten wissen wollte. Wenn Cyprian demgegenüber mehr als einer den Standpunkt der bischöflichen Würde und Macht vertreten hat, so ist es um so bemerkenswerter, daß seinem gelehrigen Schüler der alte Sprachgebrauch noch so lebendig ist. Freilich betont noch Hieronymus die Gleichheit des Presbyters und Bischofs, aber er begründet seine Behauptung mehr als Gelehrter mit dem neutestamentlichen Sprachgebrauch und verwahrt sich bezeichnenderweise dagegen, daß man dies für eine rein persön- 
liche Meinung halte, ${ }^{1}$ während Pontius das Wort in seinem alten Sinne noch völlig naiv gebraucht.

Die Taufe Cyprians und seine Wahl zum Bischof müssen unmittelbar aufeinander gefolgt sein. „Kein Verzug, kein Aufschub fand hinsichtlich der Gnade Gottes statt", sagt Pontius. "Ich habe noch zu wenig gesagt: das Priester- oder Bischofsamt hat er sofort empfangen (c. 3)." Man habe ihn für einen Neuling gehalten, aber schon in den ersten Tagen seines Glaubens sei seine edle Anlage so leuchtend hervorgetreten, daß auf seinem Antlitz zwar noch nicht die Hoffnung auf das Amt, aber doch der Glanz davon zu lesen gewesen sei, der das volle Vertrauen auf das ihm bevorstehende Priestertum verhieß (c. 5). ${ }^{2}$ Setzt man diese Rhetorik in die schlichte Sprache der Tatsachen um, so ergibt sich, daß Cyprians Taufe nach dem Tode seines Vorgängers beschleunigt wurde, damit ihr Mangel kein Hemmnis bei seiner Wahl bereite, und man wird die dann folgende Schilderung von dem demütigen Widerstande und Sträuben, das er seiner Wahl entgegensetzte, nicht allzu ernsthaft nehmen. Wir sind. gewiß nicht berechtigt, die Aufrichtigkeit der christlichen Überzeugung Cyprians und seine innere Umwandlung in Zweifel $\mathrm{zu}$ ziehen, allein damit sind die Motive eines durch diese Umwandlung veredelten Ehrgeizes sehr wohl $\mathrm{zu}$ vereinigen. Es mögen auch in letzter Stunde Schwankungen und Bedenken durch seine Seele gegangen sein, aber das schließt nicht aus, daß er seinen Übertritt zum Christentum mit dem festen Vorsatz vollzogen hatte, in der Gemeinde den Platz einzunehmen, der seinen Talenten und seinem Charakter entsprach. Nach Pontius war es das Volk, das inn zum Bischof haben wollte, während einige seiner Wahl widerstrebten. Wir wissen durch Cyprian selbst, daß dies Mitglieder des Presbyteriums waren, fünf ạn der Zahl, über die er sich in den schärfsten Ausdrücken ergeht. In der giftigen Gesinnung, die sie gegen sein Amt und die Stimme des Volkes hegten, hätten diese Verschwörer ihren alten Kampf und ihre frevelhaften Machenschaften gegen ihn erneuert (ep. 43,1). Pontius' Bemerkung hebt jeden Zweifel darüber auf, wann sie diesen Kampf geführt hatten. Ein anderer Presbyter hatte Cyprian zum Christentum bekehrt, und das Verhältnis zwischen den beiden war wie das

1 In Titum I 5, Migne P. L. 26, 597 Idem est ergo presbyter qui et episcopzes. Vgl. bes. Ep. 146 P. L. 21, $1192 \mathrm{ff}$.

2 ut etsi non dum efficii spei tamen fulgore resplendens imminentis sacerdotii totain fiduciam pollicaretur. So Hartel nach T. Harnack verwirft spei als eine Glosse zu imminentis sacerdotii. Es ist mit $w \mu^{2}$ spe zu lesen. 
eines Sohnes zum Vater geblieben (Pontius c. 4). Das Presbyterium war also vor der Wahl gespalten gewesen, und wir haben allen Grund zu glauben, daß der Widerstand der dissentierenden Presbyter nur durch das stürmische Verlangen des Volkes überwunden wurde. Die Menge belagerte Cyprians Haus und besetzte die Türen, so daß er nur durch ein Fenster hätte entschlüpfen können. Mit ängstlicher Spannung wartete sie auf sein Erscheinen und mit überschwenglicher Freude begrüßte sie ihn, als er sich dann endlich zeigte. Pontius sagt nichts von einer Beteiligung auswärtiger Bischöfe an der Wahl. Das ist freilich nur ein argumentum e silentio, aber es wird verstärkt durch ein zweites solches von stärkerem Gewicht. Wo Cyprian selbst die Rechtmäßigkeit seiner Wahl begründet, sagt er kein Wort davon, obwohl er alle Ursache hatte, keinen Rechtstitel auszulassen. Dem Märtyrer Puppianus gegenüber, der ihm im sechsten Jahre seines Episkopats die Gemeinschaft aufsagte, beruft er sich auf das Urteil und Zeugnis Gottes, der ihn zum Bischof gemacht habe. Freilich hatte Puppianus nicht seine Wahl, sondern seine sittliche Führung beanstandet. Aber gegen diese Bezichtigung beruft er sich auf die Legitimität seines Bischoftums. Seine Logik ist: Gott macht die Bischöfe, Gott aber macht keinen Unwürdigen zum Bischof. Ich bin Bischof, folglich bin ich nicht unwürdig, und wer sich gegen mich auflehnt, lehnt sich gegen Gottes Urteil und Zeugnis auf (Ep. 66,1). Dagegen nimmt er um dieselbe Zeit bei einer andern Gelegenheit den Standpunkt ein, daß die bloße Tatsächlichkeit des Amtes keineswegs seine Legitimität beweist, sondern daß zu einer gesetzmäßigen und richtigen Bestallung zwei Erfordernisse nötig seien, daß jeweilig die nächsten Bischöfe derselben Provinz zur Wahl eines Bischofs zusammenkommen und die Wahl in Gegenwart des Volkes stattfindet, dem das Leben der einzelnen genau bekannt ist. Die Ordination erfolgt de universae fraternitatis suffragio et. de episcoponum qui in praesentia convenerant iudicio (ep. 67,5). Von dem Urteil Gottes ist hierbei keine Rede, und es wird festgestellt, daß manchmal Unwürdige zu Bischöfen ordiniert werden (ep. 67,4), das Volk aber in einem solchen Falle verpflichtet ist, mit dem Bischof nicht weiter zu kommunizieren (ep. 67,3). Wenn also Cyprian sich Puppianus gegenüber hinter dem Urteil Gottes verschanzt, so muß man daraus schließen, daß er die Rechtstitel, die er ep. 67,5 von einem ordnungsmäßigen Bischof verlangt, für sich selber nicht geltend machen konnte und er daher genötigt war, sich auf ein klangvolles, aber tatsächlich völlig bedeutungsloses Argument zurückzuziehen.

Dieser Schluß wird dadurch bestätigt, daß er auch vor dem Bischof 
Cornelius die von seinen Feinden angegriffene Rechtmäßigkeit seiner Amtsführung nicht wesentlich anders verteidigt. Er sei zum Bischof an Stelle des verstorbenen bestellt, durch die Stimme des Volkes .im Frieden erwählt, durch Gottes Hilfe in der Verfolgung beschützt, allen seinen Amtsgenossen treu verbunden, dem Volke in seinem Bischofsamt vier Jahre lang erprobt (ep. 59,6). Auch hier wird der Nachdruck auf die Tatsächlichkeit seines Bischoftums gelegt, und wenn er auf den Beistand Gottes in der Verfolgung, die Verbindung mit seinen Amtsgenossen, die Anerkennung des Volkes während seiner Amtsführung hinweist, so kann das nur den Sinh haben, daß er darin den nachträglich gelieferten Beweis für die Rechtmäßigkeit seiner Wahl sieht. Hätte er sich darauf berufen können, daß er von Bischöfen eingesetzt sei, so würde er das um so weniger unterlassen haben, als er für die Gültigkeit der Wahl des Bischofs Cornelius in erster Linie den Umstand geltend macht, daß er von den in Rom anwesenden Bischöfen dazu gemacht sei. Allerdings hat Benson in dem Briefe an Cornelius ein Zeugnis für die Anwesenheit von Bischöfen bei der Wahl Cyprians zu finden geglaubt (S. 26 Anm. 3 a. E.). Aber dieses Zeugnis kann keine Gültigkeit beanspruchen. Cyprian führt c. 5 den Ursprung aller Ketzereien und Spaltungen auf den Ungehorsam gegen den Bischof zurück: Cui si secundum magisteria divina obtemperaret fraternitas universa, sagt er, nemo adversum sacerdotum collegium quicquam moveret, nemo post divinum iudicium, post populi suffragium, post coepiscoporum consensum, iudicem se non iam episcopis, sed deo faceret. Hier wird wieder ein anderer Wahlmodus vorausgesetzt als derjenige, den er ep. 67, 5 als Regel bezeichnet. Denn hiernach wird offenbar der zu wählende Bischof nicht dem Volke von den Bischöfen der Umgegend präsentiert, sondern diese stimmen der Wahl des Volkes bei, während nach ep. 67,5 die Bischöfe die Initiative, das Volk das Recht des Einspruchs oder vielmehr die Gewohnheit der Akklamation hatte. Nach den Umständen muß man annehmen, daß dieser Modus der Wahl Cyprians mehr entsprach als der andere, denn wenn die Bemerkung auch ganz allgemein aufgestellt ist, so muß man doch nach dem Zusammenhange dabei in erster Linie an Cyprian denken. Aber der Ausdruck ist so vorsichtig gewählt, daß damit keineswegs gesagt ist; daß die Zustimmung der Bischöfe unmittelbar nach der Wahl des Volkes erfolgte und diese erst dadurch ihre Gültigkeit gewann. Wenn man den Ausdruck pressen will, ist das sogar ausgeschlossen, denn von Mitbischöfen kann erst gesprochen werden, wenn derjenige, um den es sich handelt, vollgültiger Bischof geworden ist. Auch hier spenden die 
Bemerkungen über die Wahl des Cornelius ep. 55, 8 Licht. Denn auch hier wird ein consensus coepiscoporum geltend gemacht, wenn gesagt wird, man müsse sich in der Beurteilung des Cornelius an das Urteil Gottes, der den Bischof mache, und das Zeugnis der Mitbischöfe halten, deren gesamte Zahl in der ganzen Welt in einträchtiger Einmütigkeit übereingestimmt habe (concordi unanimitate consensit). Dieses Zeugnis ist wohl zu unterscheiden von dem Zeugnis, das in der Wahl des Cornelius durch die in Rom versammelten Bischöfe zum Ausdruck kam. Bei den meisten Bischöfen hat dieses natürlich nur in stillschweigender Anerkennung bestanden, und daher wird ep. 59, 5 tatsächlich nicht mehr gesagt, als daß gegen Cyprians Wahl von den Bischöfen Afrikas nicht protestiert sei, und erst im folgenden Kapitel, wo Cyprian von sich im besonderen spricht, erfolgt die positive Ergänzung, daß er mit allen Kollegen treu verbunden sei.

So werden die Grundlagen der Darstellung des Pontius durch eine Analyse der eigenen Aussagen Cyprians über seine Wahl durchaus bestätigt. Er hat die Stimme des Volkes für sich gehabt, aber nicht die Gesamtheit des Presbyteriums, geschweige denn, daß Bischöfe zu seiner Wahl mitgewirkt hätten. Darüber, ob er als Laie oder als Priester zur Bischofswürde gelangt war, äußert sich Cyprian nicht. Aber wenn wir bedenken, daß er um die Zweifel, die an der Gültigkeit der Wahl des Cornelius entstanden waren, jeden Umstand berücksichtigt, der dafür sprach, wenn er betont, daß Cornelius nicht plötzlich zu dem höchsten Gipfel des Priestertums befördert, sondern aus dem Presbyterium hervorgegangen sei, nachdem er alle Stufen der priesterlichen Laufbahn ordnungsmäßig durchlaufen hatte (ep. 55,8), so muß man annehmen, daß er dasselbe nicht für sich anführen konnte, als er sich gegen die Angriffe seiner Gegner verteidigte, die für den inzwischen gewählten Gegenbischof Fortunatus (ep. 59, 9) eintraten, obwohl, wenn er es konnte, er um so mehr Ursache hatte, es zu tun, als jene Angriffe nicht ohne Eindruck auf Cornelius geblieben waren (ep. 59, 2).

$\mathrm{Daß}$ diese Mängel seiner Wahl von seinen Gegnern nicht unberührt gelassen wurden, ist um so wahrscheinlicher, als der Ausbruch des Kampfes gegen seine Person von ihm selbst auf jene Presbyter, die seiner Wahl widerstrebt hatten, zurückgeführt wird und der Gegenbischof Fortunatus ohne Zweifel derselbe wie der ep. 14, 4 genannte Presbyter Fortunatus ist. Aber den Hauptgegenstand der Anklage haben sie sicher nicht gebildet, das waren vielmehr die gehässigen Vorwürfe sittlicher Verfehlungen (ep. 59, 2 und 66,1), mit denen die Gegner in den geistlichen Lagern genau so schnell bei der Hand waren wie einst 
in den politischen. Tatsächlich hatten jene Presbyter ebenso wie der Märtyrer Puppianus seine Wahl bis zu seiner. Flucht in der decianischen Verfolgung anerkannt. Denn der erste Brief, den er aus seinem Versteck an die Presbyter und Diakonen seiner Gemeinde richtete, ep. 5, ist in dem sichern Gefühl geschrieben, daß sein Amt während seiner Abwesenheit bei diesen in den besten Händen sei, und dem Märtyrer Puppianus hält er vor, daß er bis zur decianischen Verfolgung keinen Anstoß, mit ihm zu kommunizieren, genommen habe (ep. 66, 4). Wahrscheinlich ist also, daB, was von dem Standpunkt, den Cyprian später selbst einnahm, als Mangel an seiner Wahl erscheinen mußte, erst hinterher von seinen Gegnern geltend gemacht worden. Die Regel, die nach Cyprians Behauptung bei. der Bischofswahl in Afrika und fast allen Provinzen beobachtet wurde, daß dazu die nächsten Bischöfe zuisammenkamen und die Wahl dann in Gegenwart des Volkes vollzogen wurde (ep. 67,5), ist wohl erst zu Cyprians Zeit zu allgemeinerer Anwendung gekommen. Cyprian begründet sie durch die Berufung auf Num 20, $25 \mathrm{f}$. und Act 1, 15, nicht auf die Tradition. Ergibt sich die Abweichung von dieser Regel bei seiner Wahl aus Pontius' Darstellung und seinen eigenen Angaben nur indirekt und dürfen wir in beiden Fällen wohl eine bew̧ußte Verschleierung des Tatbestandes annehmen, so bezeichnet Pontius die Wahl eines Neophyten zum Bischof ausdrücklich als den ersten und wohl einzigen Fall in seiner Art. Das ist durchaus falsch, aber die ausdrückliche und nachdrückliche Hervorhebung der Tatsache ist für Pontius außerordentlich charakteristisch. Denn sie dient nach ihm seinem Helden zum ganz besonderen Ruhme, da sie durch die beispiellose Kraft seines jungen Glaubens gerechtfertigt sei. Hier geht die apologetische Tendenz völlig in die panegyrische auf, und vielleicht dachte auch zu der Zeit, als Pontius schrieb, niemand mehr daran, diese Unregelmäßigkeit gegen Cyprian einzuwenden.

Dagegen können die Vorwürfe, die man ihm wegen seiner Flucht in der decianischen Verfolgung machte, noch nicht verstummt gewesen sein. Er würde sonst nicht mit solchem Nachdruck versichert haben, daß Cyprian sich nicht aus Angst versteckt habe. Er begegnet diesem Vorwurf mit kecker Rhetorik. Angst sei ès schon gewesen, aber eine berechtigte, die Angst, die fürchtete, den Herrn zu beleidigen, die Angst, die lieber den Vorschriften Gottes willfahren als sich krönen lassen wollte. Denn da der Herr ihm das Versteck geboten hätte, so habe er geglaubt, wenn er nicht gehorchte, sich durch das Martyrium zu vergehen. Aber nachdem Pontius bereits in c. 7 nachgewiesen hatte, 
daß nur durch den Aufschub des Martyriums seine so notwendige schriftstellerische Tätigkeit ermöglicht worden sei, kommt er in c. 8 noch einmal auf den Nutzen dieses Aufschubs zurück, um zu beweisen, daß er nicht die Folge menschlicher Kleinmütigkeit, sondern göttlichen Ratschlusses gewesen sei. Hierbei wird denn nun auch klar, von welcher Seite jener Vorwurf noch immer wiederholt wurde. Denn nachdem Pontius auseinandergesetzt hat, wie Cyprian die Wunden der Verfolgung geheilt und das Schiff der Kirche durch die Spaltungen glücklich hindurchgeführt habe, schließt er: Non haec, oro, consilia divina sunt?. Hoc fieri sine deo potuit? Viderint qui putant posse fortuitu ista contingere. Ecclesia illis clara voce respondet dicens: Ego sine dei nutu necessarios reservari non admitto, non credo. Es war die Gegenkirche, die sich auf das Betreiben des Felicissimus in Carthago gebildet und einen eigenen Bischof gewählt hatte. Sehr wahrscheinlich war sie in das novatianische Lager übergegangen. Diese Stelle liefert den Beweis, daß sie Cyprian überlebt hatte und in ihrer alten Feindschaft beharrte. Denn die Personifikation der Kirche beruht nicht, wie Harnack meint (S. 55), auf einer bewußten oder unbewußten Nachwirkung des Hermas. Die Personifikation der Kirche im Hirten ist von ganz anderer Art, ja es ist die Frage, ob man hier überhaupt von der Personifikation eines Begriffes reden kann. In diesem Zusammenhange ist das Wort offenbar antithetisch gebraucht, die Zurechtgewiesenen gehören der Kirche nicht an. Auch Cyprian läßt ep. 59, 7 zwar nicht die Kirche, aber Petrus im Namen der Kirche (ecclesiae voce) reden, und er definiert dabei die Kirche als den Inbegriff derjenigen, die im Hause Gottes bleiben im Gegensatz zu denjenigen, die von Christus abgewichen sind, und wendet das Wort Petri Joh 6,68 auf die Verhältnisse der Gegenwart an. Man braucht ecclesia nur mit "Gemeinde" zu übersetzen, so ist eine lebendige. Vorstellung ganz unmittelbar gegeben, und wenn die Vielen, die die Gemeinde bilden, hier im Singular, statt im Plural reden, so ist das darum berechtigt, weil diese Vielheit als eine geschlossene Einheit empfunden wird.

Allein das eigentliche Argument des Pontius zu der Verteidigung Cyprians wegen seines Verhaltens in der decianischen Verfolgung ist nicht das Gebot und der Ratschluß Gottes, sondern die Freiwilligkeit seines Martyriums in der valerianischen: Ut nihil aliud excusem, ipse postmodum passus est, quam passionem utique ex more vitaret, si et ante vitasset. Wenn aber die Gegner auch nach seinem Martyrium nicht aufhörten, Cyprian wegen seiner Feigheit in dem einen Falle zu schmähen, so werden sie ihm schwerlich den Mut der Entschlossen- 
heit in dem andern zugestanden haben. Den. Beweis liefert Pontius selbst durch seine Darstellung des Martyriums, in der die apologetische Tendenz mit der panegyrischen auf das innigste verflochten ist. Dies $\mathrm{zu}$ zeigen und $\mathrm{zu}$ versuchen, über die Gunst und Ungunst der Parteien hinaus $\mathrm{zu}$ der geschichtlichen Wahrheit vorzudringen, wird die Aufgabe des Schlußkapitels sein.

\section{Ev. Joh 10, 29. 30.}

In dieser Zeitschrift hatte ich 1909 (Jahrg. X) S. 324-26 gezeigt, daß das Ziel des Verses 29 die Einheit von Vater und Sohn v. 30 ist; demnach sind alle Übersetzungen falsch, die vom Vater aussagen, er sei größer als alles, als die Schafe, als alle und was dergleichen mehr geleistet wird. Falsch ist auch das objektlose: Niemand kann aus der Hand des Vaters rauben, so $\mathrm{H}$. Holtzmann und Weizsäcker; oder: Niemand vermag sie der Hand des Vaters $z u$ entreißen, so Heitmüller. mit Luther. Vielmehr bezieht sich dieser Satz auf dasselbe Ziel wie der erste: Niemand kann die Einheit zwischen Vater und Sohn der Hand des Vaters entreißen. Diese Übersetzung läßt nicht nur der Vulgatatext zu. Pater meus (absichtlich vorangestellt), quod dedit mihi, majus omnibus est et nemo potest rapere de manu Patris mei: Ego et Pater unum sumus (cf. Aug. in Joh 48,6), sondern auch der griechische;

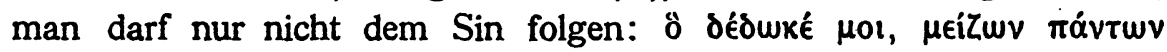

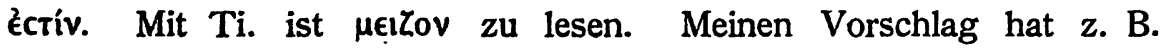
Fr. Spitta angenommen (das Johannes-Evangelium als Quelle der Geschichte Jesu 1910 S. 228-29). Aber ich habe hinterher gefunden, daß schon längst Vorgänger dagewesen sind: van Ess, Allioli und Kistemaker. Deren Übersetzungen sind mir leider nur im beschränkten Maß zugänglich. Unter den verschiedenen Ausgaben der Übersetzung des Leander van Ess kommt wohl nur die Sulzbacher $1807 \mathrm{ff}$. in Betracht. J. F. Allioli, Die heiligen Schriften des AT und NT, übersetzt Landshut $1830 \mathrm{ff}$. J. Hyac. Kistemaker, Die heiligen Schriften des NT, übersetzt Münster 1825 . Vgl. die Polỳglottenbibel von R. Stier und W. Theile ${ }^{3} 1854$ (Bielefeld).

Kiel.

Dr. Roland Schütz. 